\title{
Revisión del tratamiento del uso conjunto del tabaco y del cannabis
}

\section{Review of tobacco and cannabis co-use treatment}

Fecha de recepción: 14-01-2019

Fecha de aceptación: 13-05-2019

\author{
Ana Esteban Herrera \\ Psicóloga. Coordinadora Proyecto EVICT- evidencia cannabistabaco. \\ Eduardo Olano Espinosa \\ Médico. Servicio Madrileño de Salud. \\ José Javier Moreno Arnedillo \\ Psicólogo-Madrid Salud-Ayuntamiento de Madrid \\ Dra. Cristina Pinet Ogué \\ Psiquiatra. Hospital de la Santa Creu i Sant Pau. Barcelona. \\ María José Duaso Ansó \\ Enfermera King's College London.
}

\section{resumen/abstract:}

El consumo conjunto y paralelo de tabaco y cannabis es un fenómeno de tendencia creciente, especialmente entre los jóvenes, y que contiene importantes implicaciones clínicas que conviene clarificar a la vista de la diversidad de pautas de abordaje existentes. Se realizó una revisión bibliográfica de carácter descriptivo o cualitativo sobre el tratamiento del consumo de tabaco y cannabis. De la revisión realizada se concluyen varias recomendaciones: el abordaje conjunto de ambos consumos, la orientación a la abstinencia completa e indefinida, la individualización del tratamiento y la atención a los diversos objetivos parciales. Entre los tratamientos psicológicos, está bien establecida la efectividad de la entrevista motivacional, la terapia cognitivo-conductual y la terapia de incentivo con refuerzo comunitario. Entre los tratamientos farmacológicos, cuenta con evidencia el uso de sustitutivos de nicotina, bupropion y vareniclina para la dependencia del tabaco; en el caso del cannabis, no existen medicamentos aprobados, si bien existen líneas de trabajo interesantes con naltrexona, n-acetilcisteína, gabapentina y agonistas cannabinoides. Finalmente, los últimos años han visto una eclosión de aplicaciones móviles (mHealth) que ofrecen importantes ventajas y que han sido ya objeto de revisión sistemática.

Co-use of cannabis and tobacco whether it is co-administered or concurrent is a phenomenon of increasing tendency, especially among young people. Co-use has important clinical implications that should be clarified given the diversity of existing treatment approaches.

A narrative review of the literature on the treatment of cannabis and tobacco use was carried out. Several recommendations emerge from this review: The joint approach for both uses, the aim towards complete and indefinite abstinence, the individualization of the treatment and the importance of addressing the diverse sub-goals towards cessation. Among the psychological treatments, the effectiveness of motivational interviewing, cognitive-behavioral therapy and incentive therapy with community reinforcement is well-established. Among the pharmacological treatments, there is evidence of the use of nicotine replacement therapy, bupropion and varenicline for tobacco dependence; In the case of cannabis, there are no approved medications, although there are interesting lines of work with naltrexone, $n$-acetylcysteine, gabapentin and cannabinoid agonists. Finally, recent years have seen an explosion of mobile applications (mHealth) that offer important advantages and that have already been subject to systematic review. 


\section{palabras clave/keywords:}

Cannabis; tabaco; dependencia; tratamiento.

Cannabis; tobacco; dependence; treatment.

\section{Introducción}

El consumo conjunto y paralelo de tabaco y cannabis, un fenómeno en absoluto novedoso pero sí de tendencia creciente, es una cuestión compleja, con importantes implicaciones económicas, políticas, legales, culturales, educativas y sanitarias, hasta el punto de que, en nuestro país, ha merecido la reciente creación de un grupo de trabajo en el seno del Comité Nacional para la Prevención del Tabaquismo ('Grupo EVICT') con el objetivo de proveer de evidencia científica que sustente el desarrollo de políticas de control, prevención y asistencia sanitaria en relación con esta cuestión. Este grupo presentó en 2015 un primer informe (Comité Nacional para la Prevención del Tabaquismo, 2015) del que ha derivado posteriormente diversa documentación técnica y divulgativa (disponible en www.evictproject.org) así como iniciativas de formación en distintos ámbitos.

Inevitablemente, entre los objetivos de este grupo de trabajo se incluyen la revisión de la evidencia disponible y la posterior elaboración de recomendaciones sobre el abordaje clínico del consumo conjunto de tabaco y cannabis.

Esta cuestión no ha recibido, ciertamente, la atención que merece, al menos hasta hace pocos años. Sigue siendo frecuente que, en los dispositivos de deshabituación de drogas ilegales, la atención a los consumidores de cannabis que, además, fuman tabaco (mezclado con el cannabis en forma de porro, y también de forma paralela) se dirija exclusivamente al consumo de cannabis y no del tabaco, por considerar que aquella es la 'droga principal', ignorando así las sinergias e implicaciones mutuas que existen entre ambos consumos y excusando, de modo incomprensible, el riesgo sanitario que implica el consumo de tabaco, un riesgo de magnitud tal que la mortalidad por causas relacionadas con el consumo de tabaco en pacientes tratados por dependencia de alcohol, cannabis u otras drogas es más elevada que la debida a la propia sustancia motivo de consulta (Hurt, Offord y Croghan, 1996). También es frecuente la situación contraria: policonsumidores que demandan atención para dejar de fumar tabaco y solo tabaco, y ante los cuales el profesional no cuenta con criterios de actuación claros en relación con la conveniencia o necesidad de abandonar ambos consumos ni de cómo hacerlo en caso de considerarlo. Existe evidencia de que en consumidores de cannabis que solicitan tratamiento se produce un peor resultado si no cesa el consumo de tabaco, y, a la inversa, de que la cesación de tabaco se ve interferida negativamente por el mantenimiento del consumo de cannabis (Gourlay, Forbes, Marriner, Pethica y McNeil, 1994).

El objetivo de este artículo es presentar las principales evidencias científicas identificadas en relación al tratamiento del consumo dual de tabaco y cannabis. 


\section{Material y método}

Se realizó una revisión bibliográfica de carácter descriptivo o cualitativo sobre el tratamiento del consumo de tabaco y cannabis. Para las diferentes búsquedas se utilizaron varias bases de datos: Scopus, PubMed y Proquest (agrupa un conjunto de bases de datos), combinando términos relacionados con ambas sustancias y su tratamiento. Para la sección de intervenciones en contextos no clínicos se realizaron búsquedas en las bases de datos: Embase, Web of Science, Medline, PsychINFO y CINAHL, utilizando una combinación de términos relacionados a tabaco, cannabis y mheallth. La última búsqueda se realizó en diciembre 2018.

\section{Resultados}

De la revisión realizada y de otras posteriores de Gates, Sabioni, Copeland, Le Foll y Gowing (2016), así como de las recomendaciones del National Institute on Grug Abuse norteamericano (NIDA, 2010) cabe sintetizar las siguientes orientaciones para el tratamiento del consumo y la dependencia de tabaco y cannabis:

\section{A. Sobre la estrategia de abordaje de ambos consumos}

Con independencia de que no coincida con la práctica asistencial habitual, se recomienda el abordaje conjunto de ambos consumos y, por lo tanto, plantear siempre esta opción como preferible al usuario, aunque no sea esa su pretensión inicial.

Esta recomendación se basa en la fuerte asociación entre ambos consumos (misma vía de administración, contextos y funcionalidad similares...) (Bélanger, Akre, Kuntsche, Gmel y Suris, 2011; Hublet et al., 2015; Peters, Budney y Carroll, 2012; Rabin y George, 2015; Ramo, Liu y Prochaska, 2012) y, por lo tanto, en los peores resultados que se obtienen cuando se busca la cesación del consumo de una de las sustancias sin modificar el de la otra (Agrawal, Budney y Lynskey, 2012; Amos, Wiltshire, Bostock, Haw y McNeill, 2004; Nieva, Ortega, Mondon, Ballbé y Gual, 2010).

\section{B. Sobre los objetivos terapéuticos}

Se recomienda un objetivo de abstinencia completa e indefinida de ambas sustancias, que puede abordarse de forma simultánea o sucesiva.

Este objetivo es complejo e implica su división en varios objetivos parciales; cada uno de ellos tiene entidad propia y requiere procedimientos y estrategias independientes y específicos para su consecución, tal como veremos en el apartado dedicado a los tratamientos psicológicos.

1. Construcción de la motivación y toma de decisiones, negociación de objetivos y clarificación de expectativas

Antes de abordar la cesación de ambos consumos, es crítica la optimización de la motivación o disposición del consultante para ello. Más aún cuando a menudo este objetivo no coincide con su intención inicial. De nada sirve disponer de tratamientos efectivos si el beneficiario potencial no está dispuesto a utilizarlos. Es básico que el profesional esté entrenado en los principios y las estrategias de la entrevista motivacional. 
En este mismo contexto deben clarificarse los objetivos de la intervención en relación con el consumo (abstinencia total e indefinida) y el plan de objetivos intermedios o auxiliares (aprendizaje de habilidades, rediseño del contexto, cambios de estilo de vida...). En este momento inicial es necesario dedicar tiempo a identificar y clarificar determinadas expectativas inadecuadas que, por serlo, no se cumplen y pueden generar frustración y abandono (sobre la naturaleza del proceso de deshabituación, sobre su dificultad, sobre la importancia del compromiso activo, sobre las posibles recaídas, sobre el afrontamiento del deseo de consumir, sobre los ingredientes del proceso...).

\section{Cesación del consumo}

Se negocia un día para dejar completamente de consumir. Implica el uso correcto de los apoyos farmacológicos y la planificación cuidadosa de los entornos estimulares durante los primeros días sin consumir.

\section{Prevención y afrontamiento de las recaídas}

La recaída o incumplimiento de propósitos es un fenómeno que forma parte del proceso de deshabituación, un proceso complejo que supone el abandono de hábitos sumamente arraigados y con importante valor instrumental. Aunque habitualmente este objetivo se acomete justo después de la cesación, es cualitativamente diferente y las estrategias para conseguirlo son independientes por completo de los procedimientos que se hayan seguido para la interrupción del consumo.

Se trata aquí de dos cuestiones diferentes: por un lado, prevenir la recaída; por otro, prever cómo afrontarla en caso de que se produzca.

\section{Abordaje de problemas asociados}

El plan de tratamiento debe contemplar actuaciones dirigidas a otros comportamientos problemáticos o circunstancias personales asociadas al consumo (depresión, ansiedad, dificultades sociales, consumo de otras sustancias...), lo cual puede requerir la coordinación con otros recursos intra o extrasanitarios.

\section{Sobre los contenidos terapéuticos}

El plan de intervención debe construirse a la medida del caso, a partir de la hipótesis elaborada sobre las variables que mantienen el consumo, y dirigido a los objetivos señalados en el apartado anterior. No existe un solo tratamiento que sea apropiado para todas las personas (NIDA, 2010). El uso de etiquetas diagnósticas similares puede llevar al error de creer que existe una correspondencia unívoca entre la etiqueta diagnóstica y el tratamiento indicado, cuando en realidad se trata de problemas complejos donde intervienen factores personales, biográficos y contextuales diversos y que operan de modo diferente entre diferentes individuos, aunque la morfología del comportamiento sea similar.

\section{Tratamiento psicológico}

Los principales tratamientos psicológicos cuya efectividad ha sido bien establecida son la entrevista motivacional, la terapia cognitivo conductual y la terapia de incentivo con refuerzo comunitario (Lévesque y Le Foll, 2018; Sabioni y Le Foll, 2018; Schwitzer et al., 2016; 
Sherman y McRae-Clark, 2016; Walther, Gantner, Heinz y Majic, 2016; Davis et al., 2015; Lee et al., 2014; Ramo, Liu y Prochaska, 2012; NIDA, 2010; Becoña y Cortés, 2008; Dutra et al., 2008).

El metaanálisis de Dutra et al. (2008) concluye que los tamaños del efecto encontrado son notables para los tratamientos de manejo de contingencias, prevención de recaídas y terapia cognitivo conductual. Davis al. (2015) en un metaanálisis sobre intervenciones conductuales en la dependencia de cannabis, concluyen igualmente que el manejo de contingencias, la prevención de recaídas, la entrevista motivacional y la terapia cognitivo conductual son significativamente superiores a las condiciones de control. En esta misma línea van igualmente las recomendaciones del National Institute on Drug Abuse (NIDA, 2010) y las conclusiones de las revisiones de Lee et al. (2014), de Ramo et al. (2012) y Becoña y Cortés (2008). Similares resultados encuentran Schwitzer et al. (2016), Sherman et al. (2016), Walther et al. (2016), Lévesque y Le Foll (2018) y Sabioni y Le Foll (2018).

Por otra parte, y tal como señalábamos en la introducción, el tratamiento integrado de la dependencia de tabaco y cannabis incluye varios objetivos parciales y secuenciales. Cada uno de ellos tiene entidad propia y su consecución es necesaria para abordar el siguiente. Cabe ordenar los tratamientos psicológicos en función del objetivo parcial al que se dirigen, según se representa en la figura 1 .

Figura 1.- Objetivos parciales y procedimientos del tratamiento psicológico

\section{Objetivos}

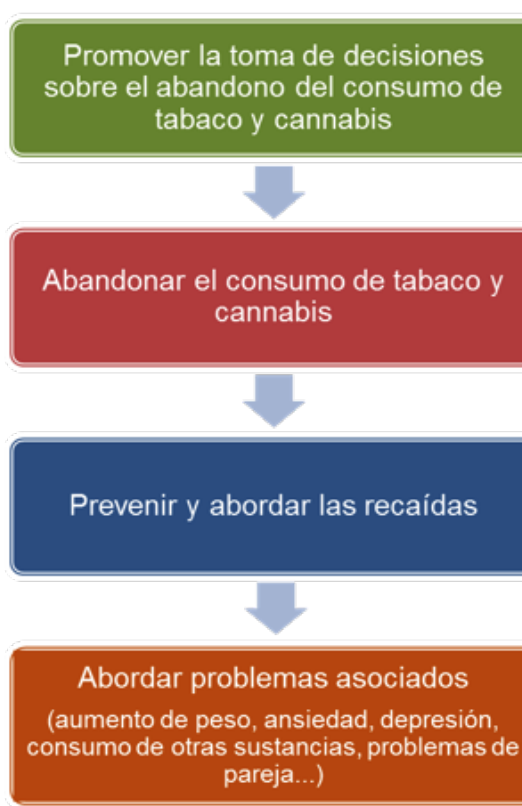

\section{Técnicas}

Establecimiento de relación de colaboración / Entrevista Motivacional / Información y modificación de expectativas / Balance decisional / Establecimiento de objetivos y plan de tratamiento
Técnicas de reducción gradual / Técnicas aversivas / Tratamiento farmacológico / Pautas de control estimular
Técnicas de exposición al estímulo/ Planificación de cambios de estilo de vida y activación conductual/ Entrenamiento autoinstruccional/ Entrenamiento en habilidades: relajación, resolución de problemas, competencia social / Afrontamiento de los consumos puntuales, las ambivalencias y las recaídas / Terapia de incentivo con refuerzo comunitario
Tratamiento conductual especifico e individualizado 
Dado que en numerosas ocasiones el paciente no desea o se muestra ambivalente ante la posibilidad del abandono del consumo de tabaco y cannabis, o su deseo se orienta al abandono del consumo de una de las dos sustancias pero no de la otra (dejar el tabaco pero seguir fumando cannabis, o al revés), es de suma importancia el uso de técnicas de entrevista motivacional y, en general, de todas aquellas estrategias de comunicación que faciliten el acuerdo en los objetivos terapéuticos entre usuarios y profesionales. La entrevista motivacional es una estrategia básica porque la motivación del consultante es fuertemente contextual. Las características del comportamiento del consultor, influyen en la decisión y el grado de compromiso del consultante en un proceso complejo. La entrevista motivacional implica el establecimiento de una relación interpersonal de colaboración, y puede incluir la clarificación de determinadas expectativas erróneas sobre el tratamiento y la negociación de objetivos. En caso de que el usuario rechace el tratamiento simultáneo, cabe plantear un abordaje sucesivo (Peters et al., 2012) puesto que lograr la deshabituación del consumo de una sustancia suele funcionar como elemento motivador que favorece la decisión el abandono de la otra (Lee et al., 2014).

Asimismo, la prevención y el abordaje de las recaídas y dificultades una vez conseguida la abstinencia es un objetivo con entidad propia y que implica procedimientos de aprendizaje de habilidades, modificaciones del estilo de vida, rediseño del contexto, técnicas de exposición y afrontamiento de los consumos puntuales.

Finalmente, cabe mencionar una de las terapias psicológicas de tercera generación con prometedores resultados: la Terapia de Aceptación y Compromiso (Hayes, Strosahl y Wilson, 1999). Aunque existe evidencia en el ámbito de las adicciones para esta terapia se hace necesario seguir investigando, no sólo para lograr un mayor cuerpo empírico, sino para detectar aspectos claves del tratamiento y mejorar las intervenciones (López HernándezArdieta, 2014).

\section{Tratamiento farmacológico}

En referencia al abordaje farmacológico, los pacientes con consumo conjunto de tabaco y cánnabis suelen estar excluidos de los ensayos clínicos por lo que no hay muchas evidencias de calidad.

Sin embargo, el tratamiento de primera línea para la dependencia a la nicotina (sustitutivos de nicotina, bupropion, vareniclina) podrá utilizarse en los consumidores conjuntos con las mismas pautas que en el caso de la nicotina sola salvo excepciones (Baca, 2009).

La terapia sustitutiva con nicotina ha sido la primera estrategia farmacológica que ha demostrado evidencia en el tratamiento del tabaquismo (Fiore et al., 2008). Las formas de presentación disponibles en nuestro país son las formas de liberación lenta en forma de parches (dosis de 21, 14 y $7 \mathrm{mg} / 24 \mathrm{~h} ; 15$ y $10 \mathrm{mg} / 16 \mathrm{~h}$ ), y las formas de liberación rápida en forma de chicles (2 y $4 \mathrm{mg}$ ), comprimidos para chupar (1, 1,5,2 y $4 \mathrm{mg}$ ) y espray bucal (1 $\mathrm{mg}$ /pulverización). En general se recomienda la utilización de parches con una dosificación estándar durante 8-12 semanas para mantener una liberación constante de nicotina durante el día y el uso de sustitutivos de liberación rápida para el alivio puntual de la ansiedad y el 
deseo de fumar si fuera necesario. El inicio del tratamiento, salvo en casos especiales, es en el día que se abandona el consumo de tabaco.

El bupropion es un antidepresivo que actúa sobre las vías noradrenérgicas y dopaminérgicas, así como sobre los receptores nicotínicos. Se comercializa en comprimidos de $150 \mathrm{mg}$. La pauta estándar recomendada se inicia a dosis de $150 \mathrm{mg}$ durante 7 o 10 días y posteriormente se sube a $300 \mathrm{mg}$, momento en el cual se deja de fumar. Es un medicamento bien tolerado, pero con algunos efectos adversos, como nauseas, cefalea, boca seca e insomnio, como más frecuentes. En fumadores de cannabis además se ha informado de alteraciones del estado de ánimo.

Finalmente, la vareniclina actúa como un agonista parcial selectivo de los receptores nicotínicos. Su acción antagonista puede bloquear el efecto de la nicotina en los receptores disminuyendo la satisfacción del uso del tabaco. Como tratamiento único es el fármaco con mayor eficacia (Olano et al., 2011). Se comercializa en comprimidos de 0,5 y $1 \mathrm{mg}$, iniciándose el tratamiento con $0,5 \mathrm{mg}$ y escalonándose el aumento hasta la dosis de mayor eficacia de $1 \mathrm{mg} / 12 \mathrm{~h}$. Es a partir de esa dosis entre el 7 y 10 día del inicio en que se abandona el consumo de tabaco. Los efectos adversos más frecuentes son las náuseas, la alteración del sueño y la flatulencia.

El caso del cannabis es diferente, al no haber medicamentos aprobados para su uso, porque ninguno de los estudiados ha demostrado ser efectivo de forma consistente, aunque algunos estudios abrirían líneas de trabajo interesantes con naltrexona, n-acetilcisteína, gabapentina, y agonistas cannabinoides como el delta 9 tetrahidrocannabinol oral, pero se requieren más estudios (Marshall, 2014).

Hay autores que recomiendan con la evidencia actual, añadir tratamiento con gabapentina en adultos o n-acetilcisteína en adolescentes o niños como adyuvante a la terapia psicológica en aquellos pacientes que sean incapaces de mantener la abstinencia con este tratamiento (Gorelick, 2018). Estos son los dos fármacos que han mostrado resultados más favorables hasta el momento.

Sobre la n-acetilcisteína se han publicado dos ensayos clínicos en fase II, ambos con dosis de $2.500 \mathrm{mg}$ al día, en comparación a placebo y en combinación con tratamiento psicológico. Uno de ellos (116 adultos de 15 a 21 años) demuestra la mayor eficacia del fármaco en la consecución de tests de orina negativos al delta-9-tetrahidrocannabinol (THC) durante el tratamiento (odds ratio $=2,4$, IC 95\% 1,1-5,2) y al terminar éste (41 versus 27\%), así como la tasa de abstinencia a las dos semanas (36,2 versus 20,7\%). El otro ensayo (302 adultos de 18 a 50 años) no muestra ninguna diferencia. En ambos ensayos, la tolerancia al fármaco fue buena.

La gabapentina basa su eficacia en los resultados de un único ensayo clínico donde se usa como adyuvante de la psicoterapia en comparación a placebo y psicoterapia sobre 50 adultos, a dosis de $1.200 \mathrm{mg}$ diarios. Los pacientes de la rama de la gabapentina tenían menos tests positivos de orina a cannabinoides, más abstinencia autodeclarada, menos síntomas de abstinencia medidos con el Marijuana Withdrawal Checklist y una mejora mayor en los tests cognitivos. 
Por otro lado, la evidencia existente indica que los antidepresivos ISRS (inhibidores de la recaptación de la serotonina), los antidepresivos de acción combinada, los antidepresivos atípicos (bupropion), los ansiolíticos (buspirona) y los inhibidores de la recaptación de noradrenalina (atomoxetina) probablemente son de poco valor en el tratamiento de la dependencia del cannabis. En el caso del bupropion, además, se describen alteraciones del estado de ánimo en personas fumadoras de cannabis.

En el caso de vareniclina, hay publicada una serie de casos en usuarios de tabaco y cannabis donde se ve una disminución del consumo de cannabis y de la sensación de disfrute del mismo (Newcombe, 2015) y un estudio piloto donde se informa de disminución en craving a cannabis, cantidad de tabaco y cantidad de cannabis consumidos.

Morgan (2013) encuentra una interesante relación entre la utilización a demanda de cannabidiol (CBD) en 24 sujetos motivados para dejar de fumar tabaco. Este estudio piloto, de doble ciego, muestra una disminución del $40 \%$ del consumo de tabaco a la semana de tratamiento en los sujetos que usaron el CBD (ninguna disminución en el grupo placebo), sin aumento del craving de tabaco. Los autores hipotetizan un efecto del CBD sobre el receptor CB1 (Receptor endógeno de cannabinoides). El CBD es un componente no psicoactivo de la planta del cannabis con acción antagonista sobre los receptores CB1 y CB2 que está siendo estudiado por sus efectos como ansiolítico y antipsicótico.

Sin embargo, el tratamiento con agonistas, bajo la perspectiva del éxito obtenido en el tratamiento de la dependencia a nicotina y opioides, no ha demostrado eficacia en el tratamiento del trastorno por uso de cannabis. Dos pequeños ensayos con THC sintético en combinación con lofexidina (un agonista alfa adrenérgico) no demostraron eficacia en las tasas de abstinencia, aunque si en la reducción de los síntomas de abstinencia. Otro ensayo con nabilona tuvo un resultado similar (Gorelick, 2018).

Aún más recientes son los estudios sobre la utilidad del topiramato en consumidores de cannabis. El topiramato se plantea como un fármaco prometedor, actuando a través de varios mecanismos de acción. Hay cierta evidencia de que disminuye el efecto reforzador del cannabis vía la facilitación de la transmisión del ácido g-aminobutírico y la inhibición de la transmisión glutamatérgica. Hay resultados positivos en un estudio en adolescentes y adultos jóvenes en el que el grupo que recibe tratamiento motivacional y topiramato presenta mejores resultados que el grupo placebo, tanto en cesación completa como en reducción del consumo. Uno de los problemas para poder valorar su eficacia es la alta tasa de pérdidas por efectos adversos que se halla en este estudio (Miranda et al., 2017).

El abandono del cannabis puede dar lugar a un síndrome de abstinencia (típicamente, irritabilidad, ansiedad, síntomas depresivos y/o alteración del sueño en la primera semana, normalmente leves y de resolución espontánea) que puede dificultar el mantenimiento de la misma. Si es más intenso y prolongado, o afecta a las actividades diarias, podría considerarse su tratamiento. No hay estudios que cuantifiquen el nivel de intensidad del síndrome de abstinencia a partir del cual el paciente se beneficia de estos tratamientos (Gorelick, 2018).

El tratamiento con dronabinol (delta-9-tetrahidrocannabinol sintético) demostró reducir estos síntomas y mejorar el mantenimiento de la abstinencia en un ensayo clínico, aunque no 
afectó al número de recaídas (Levin, 2011). También nabiximols, y gabapentina han demostrado reducir estos síntomas en ensayos clínicos en proporción similar, aunque no hay estudios que comparen su eficacia entre sí (Copersino, 2006). Sin embargo, como aquellos pacientes que tomen dronabinol y nabiximols pueden dar positivo a cannabinoides en el test de orina, la gabapentina puede ser una opción razonable en aquellos que queramos monitorizar mediante dichos tests.

\section{Intervenciones en contextos no clínicos}

La eclosión de las tecnologías de la información y comunicación, como internet, las tablets o los teléfonos inteligentes, abren nuevas perspectivas para desarrollar tratamientos a distancia y con menor intervención del profesional. La salud móvil, también conocida como $m$ Health, se define como la práctica de la medicina y la salud pública soportada por dispositivos móviles como teléfonos, dispositivos de monitorización de pacientes, asistentes personales digitales y otros dispositivos inalámbricos (WHO, 2011). Su evolución y desarrollo ha sido imparable en los últimos años. Hoy se pueden encontrar más de 350.000 apps de $m$ Health disponibles en diferentes plataformas, tales como Android y iOS.

Entre las ventajas potenciales de la mHealth destacan su ubicuidad, eficiencia, acceso a grandes segmentos de la población, posibilidad de personalizar o dirigir los mensajes, de automatizarlos, de actuar 24 horas al día y 365 días al año, su inmediatez y la posibilidad de contacto entre iguales para aumentar el soporte y recursos sociales.

Sus inconvenientes, su variable calidad y escaso control, ya que muchas veces están diseñadas sin relación con la evidencia existente. A nivel europeo y nacional existe regulación para la clasificación y cualificación de apps (Directiva 93/42/CE) y su correspondiente normativa española (RD 1591/2009). De acuerdo a esta normativa, si la aplicación ayuda al diagnóstico de una enfermedad, facilita el seguimiento de una condición, apoya un tratamiento, o sirve para prevenir una enfermedad, estamos hablando de un producto sanitario y por lo tanto, deben contar con el correspondiente marcado CE. Aunque muchas de las apps disponibles serían susceptibles de ser consideradas como tal, la mayoría no presenta ningún sello de calidad.

En el tratamiento del tabaquismo hay amplia experiencia en el uso de aplicaciones de salud, aunque la rápida progresión de la tecnología y el cambio de usos de la misma genera una rápida obsolescencia de los métodos empleados.

Si nos centramos en la bibliografía específicamente dirigida a conocer la eficacia del empleo de intervenciones de ayuda para dejar de fumar a través del teléfono móvil, la revisión sistemática de la colaboración Cochrane (Whittaker et al., 2016) incluye 12 ensayos clínicos que agrupan a 11.885 pacientes, con un índice de riesgo relativo combinado de 1,67 (IC 95\% 1,46-1,90) para la abstinencia a 26 semanas comparado con el grupo control, y de 1,83 (IC 95\% 1,54-2,19) con sólo los datos de los seis ensayos con validación bioquímica de la abstinencia. La mayoría de las intervenciones están basadas en modelos teóricos. Las condiciones de control son muy dispares, desde no hacer nada hasta intervenciones más intensas incluyendo ayuda farmacológica. Cabe destacar que los ensayos incluidos en este 
metaanálisis se basan en programas de intervención con mensajes multimedia (SMS) e intervenciones combinadas de internet y telefonía, pero no se encontró ningún ensayo clínico publicado o en fase de desarrollo que estudiase la efectividad de una aplicación para móvil o tableta inteligente (app), a pesar de su proliferación.

La revisión sistemática más reciente sobre intervenciones digitales y cesación tabáquica incluye 108 ensayos clínicos y 110.372 fumadores (Do et al., 2018). En comparación con los grupos de control no activos, las intervenciones para dejar de fumar que utilizan la plataforma de salud móvil ( $m$ Health) o a través de internet dieron como resultado una abstinencia de fumar significativamente mayor RR 2,03 (IC 95\% 1,7-2,03) y RR 1,71 (IC 95\% $1,35-2,16)$, respectivamente. Los investigadores resaltan que las magnitudes de los tamaños de efecto de las intervenciones son mayores si el ensayo se llevó a cabo en Europa o Estados Unidos y cuando la intervención incluyó mensajes de texto personalizados. Esto concuerda con las conclusiones de las revisiones mencionadas anteriormente y reafirma la mayor eficacia de aquellas aplicaciones que personalizan la intervención.

En cannabis el volumen de bibliografía que aborda el uso de mHealth para la deshabituación es menor que en tabaco, pero aun así encontramos tres metanálisis que han estudiado el efecto de intervenciones digitales en su consumo (Spijkerman et al., 2013; Hoch et al., 2016; Olmos et al., 2018). En conjunto incluyen un total de 15 estudios y apuntan a un efecto significativo aunque modesto en la reducción del consumo de cannabis, si bien la condición de control es variable, las intervenciones son heterogéneas y la mayoría de resultados son autorreferidos y de corta duración (menor de 6 meses).

A juzgar por el número creciente de protocolos de ensayos clínicos registrados en bases de datos internacionales, se prevé un aumento en las publicaciones de estudios que utilizan mHealth para el abordaje del consumo de tabaco y/o cannabis. Innovaciones a destacar son el uso de inteligencia artificial para el consejo de cesación tabáquica a través de BOT conversacional o el uso de Evaluación Ecológica Momentánea o EMA por sus siglas en inglés (Ecological Momentary Assessment). Esta última se refiere a la evaluación de síntomas, estados y comportamientos en un momento determinado y en el contexto cotidiano del usuario. Este tipo de aplicaciones puede facilitar la comprensión de los mecanismos que han facilitado al usuario dejar de fumar con éxito, así como los factores implicados en la recaída.

\section{Discusión}

Este artículo ha revisado la evidencia científica disponible en relación al tratamiento del consumo dual de tabaco y cannabis. La literatura revisada sugiere una mayor efectividad del tratamiento conjunto de las dos sustancias, lo cual va en contra de la práctica habitual y del deseo manifestado en la mayoría de los casos por los usuarios.

Se ha demostrado la efectividad del tratamiento psicológico, en particular de la entrevista motivacional, la terapia cognitivo-conductual y la terapia de incentivo con refuerzo comunitario. El plan de tratamiento debe ser diseñado a la medida del caso, tomando en consideración la variedad de variables que condicionan el consumo, y que pueden ser diferentes entre individuos. No existe 'un' tratamiento o una combinación de tratamientos que sean 
válidos para todos los casos. Además, la cesación del consumo implica un objetivo previo, con entidad propia, de toma de decisiones, y otro posterior, también con entidad propia, de prevención y manejo de recaídas.

El mantenimiento de la abstinencia puede requerir una ampliación de los objetivos terapéuticos y plantear cambios en el estilo de vida general del individuo, más allá de la supresión del consumo, así como intervenciones en los contextos donde se desarrolla su vida. Por lo mismo, es importante la coordinación con otros recursos (sociales, educativos, laborales, de ocio....).

Tanto el consumo de cannabis como el de tabaco están fuertemente condicionados por aspectos contextuales como la accesibilidad, precio, discurso social, existencia de modelos, manejo familiar de normas, acceso a la información, etc. Todo ello, a su vez, depende del tipo de regulación existente. El trabajo clínico no debe perder de vista esta situación.

En contraste con los tratamientos psicológicos, donde está bien establecida la efectividad de varias terapias, entre los tratamientos farmacológicos cuenta con evidencia el uso de sustitutivos de nicotina, bupropion y vareniclina para la dependencia del tabaco; Sin embargo, en el caso del cannabis, no existen medicamentos aprobados, si bien existen líneas de trabajo interesantes con naltrexona, n-acetilcisteína, gabapentina y agonistas cannabinoides. No hemos encontrado evidencias en cuanto al tratamiento conjunto, aunque el tratamiento de primera línea para la dependencia a la nicotina podría utilizarse en los consumidores conjuntos con las mismas pautas que en el caso de la nicotina sola, salvo excepciones.

En cuanto a intervenciones en contextos no clínicos, hemos destacado el protagonismo creciente de las aplicaciones digitales para el tratamiento del consumo de tabaco y cannabis. Las revisiones sistemáticas existentes sugieren una eficacia prometedora y potencial para acceder a mayores segmentos de la población pero es importante que la evidencia generada en los ensayos clínicos se traslade a las aplicaciones disponibles en el mercado y que existan regulaciones y guías que permitan al usuario elegir aquellas de mayor calidad.

En conclusión, idealmente, convendría ofrecer un tratamiento simultáneo (tabaco y cannabis a la vez). Este planteamiento implica resaltar la conveniencia de dejar de consumir cannabis a aquellas personas consumidoras de tabaco, y viceversa. Si esto no fuera posible o conveniente, puede plantearse el abandono secuencial o sucesivo (primero de una sustancia y posteriormente de la otra). Se requieren más estudios que consoliden la evidencia disponible sobre el abordaje conjunto de ambas sustancias.

\section{Agradecimientos}

Los autores agradecen al Comité Nacional para la Prevención del Tabaquismo (CNPT) el impulsar el Proyecto ÉVICT, así como la financiación de la Delegación del Gobierno para el Plan Nacional Sobre Drogas. También agradecen al grupo de trabajo ÉVICT su colaboración y en especial a los documentalistas científicos que realizaron las búsquedas bibliográficas. 


\section{Referencias}

Agrawal, A., Budney, A.J., Lynskey, M.T. (2012). The co-occurring use and misuse of cannabis and tobacco: A review. Addiction 107(7):1221-1233.

Amos, A., Wiltshire, S., Bostock, Y., Haw, S., McNeill, A. (2004). 'You can't go without a fag... you need it for your hash' - a qualitative exploration of smoking, cannabis and young people. Addiction 99:77-81

Baca, C.T. y Yahne, C.E. (2009). Smoking cessation during substance abuse treatment: what you need to know. J Subst Abuse Treat, 36: 205-19.

Becoña, E. y Cortés, M. (eds). (2008). Guía clínica de intervención psicológica en adicciones. Guías clínicas Socidrogalcohol basadas en la evidencia científica. Valencia: Socidrogalcohol. Disponible en http:// www.emcdda.europa.eu/attachements.cfm/att_231208_EN_ESO3_GuiaClinicalntPsicologica. pdf

Bélanger, R. E., Akre, C., Kuntsche, E., Gmel, G., Suris, J. C. (2011). Adding tobacco to cannabis: its frequency and likely implications. Nicotine Tob Res 13:746-750.

Comité Nacional para la Prevención del Tabaquismo (2015). Proyecto EVICT. Informe 2015. Disponible en http:// evictproject.org/wp-content/uploads/2015/09/Informe_EVICT_2015.pdf

Copersino ML, Boyd SJ, Tashkin DP, Huestis MA, Heishman SJ, Dermand JC, et al. (2006). Cannabis withdrawal among non-treatment-seeking adult cannabis users. Am J Addict, 15:8

Davis, M.L., Powers, M.B., Handelsman, P., Medina, J.L., Zvolensky, M., Smits, J.A. (2015). Behavioral Therapies for Treatment-Seeking Cannabis Users: A Meta-Analysis of Randomized Controlled Trials. Evaluation y the Health Professions, 38(1), 94-114.

Do, H.P., Tran, B.X.., Le Pham, Q., Nguyen, L.H., Tran, T.T., Latkin, C.A., Dunne, M.P., Baker, P.R. (2018) Which eHealth interventions are most effective for smoking cessation? A systematic review. Patient Preference and Adherence. 12(8), 2065-2084.

Dutra, L., Stathopoulou, G., Basden, S. L., Leyro, T. M., Powers, M. B., Otto, M. W. (2008). A meta-analytic review of psychosocial interventions for substance use disorders. American Journal of Psychiatry, 165, 179-187.

Fiore, M.C., Jaén, C.R., Baker, T.B., et al. (2008). Treating tobacco use and dependence: 2008 Update - Clinical Practice Guideline. Rockville MD: USDHHS Public Health Service.

Gates, P. J., Sabioni, P., Copeland, J., Le Foll, B., Gowing, L. (2016). Psychosocial interventions for cannabis use disorder. Cochrane Dabatase of Systematic Reviews, Issue 5, art. No. CD005336.

Gorelick, D.A. (2018). Treatment of cannabis use disorder in adults, Uptodate Topic 7796 Version 26.O. Disponible en https://www-uptodate-com.bvcscm.a17.csinet.es

Gourlay, S. G., Forbes, A., Marriner, T., Pethica, D., McNeil, J. J. (1994). Prospective study of factors predicting outcome of transdermal nicotine treatment in smoking cessation. BMJ 309: 842-6.

Hublet, A., Bendtsen, P., de Looze, M., Fotiou, A., Donnelly, P., Vilhjalmsson, R., Baska, T., Aasvee, K., Franelic, I., Gabhainn, S., Bogt, T. (2015). Trends in the co-occurrence of tobacco and cannabis use in 15-year-olds from 2002 to 2010 in 28 countries of Europe and North America. The European Journal of Public Health 25 (suppl 2): $73-75$.

Hayes, S.C., Strosahl, K.D., y Wilson, K.G. (1999). Acceptance and commitment therapy. Nueva York: The Guilford Press.

Hoch, E., Preuss, U.W., Ferri, M., Simon R. (2016) Digital Interventions for Problematic Cannabis Users in Non-Clinical Settings: Findings from a Systematic Review and Meta-Analysis.European Addiction Research, 22, 233-242.

Hurt, R. D., Offord, K. P., Croghan, I. (1996). Mortality following inpatient addictions treatment. Role of tobac- co use in a community-based cohort. JAMA 275: 1097-103.

Lee, D.C., Budney, A.J., Brunette, M.F., Hughes, J.R., Etter, J.F., Stanger, C (2014). Treatment models for targeting tobacco use during treatment for cannabis use disorder: Case series. Addictive Behaviors 39:1224-1230. 
Lévesque, A., Le Foll, B. (2018). When and How to Treat Possible Cannabis Use Disorder. Med Clin North Am, 102(4), 667-681. Disponible en 10.1016/j.mcna.2018.02.009

Levin, F.R., Mariani, J.J., Brooks, D.J., Pavlicova, M., Cheng, W., Nunes, E.V. (2011). Dronabinol for the treatment of cannabis dependence: a randomized, double-blind, placebo-controlled trial. Drug A/cohol Depend 2011, 116:142.

López Hernández-Ardieta, M. (2014). Estado actual de la terapia de aceptación y compromiso en adicciones. Salud y drogas, 14 (2), 99-108. Disponible en http://www.redalyc.org/articulo.oa?id = 83932799002

Marshall, K., Gowing, L., Ali, R., Le Foll, B. (2014). Pharmacotherapies for cannabis dependence. Cochrane Database of Systematic Reviews, Issue 12. Art. No.: CD008940. DOI: 10.1002/14651858.CD008940.pub2

Miranda, R.J., Treloar, H., Blanchard, A., Justus, A., Monti, P.M., Chun, T. et al. (2017). Topiramate and motivational enhancement therapy for cannabis use among youth: a randomized placebo-controlled pilot study. Addict Biol.,22:779-790.

Morgan, C.J.A., Das, R.K., Joye, A., Curran, H.V., Kamboj, S.K. (2013). Cannabidiol reduces cigarette consumption in tobacco smokers: Preliminary findings. Addictive Behaviors, 38:2433-2436.

Newcombe, D.A.L., Walker, N., Sheridan, J., Galea, S., Moore, T.H., Zammit, S. et al. (2015). The Effect of Varenicline Administration on Cannabis and Tobacco Use in Cannabis and Nicotine Dependent Individuals - A Case-Series. J Addict Res Ther, 6:2.

NIDA - National Institute on Drug Abuse (2010). Principios de tratamientos para la drogadicción. Una guía basada en investigaciones. U. S. Department of Health and Human Services. Disponible en https://d14rmgtrwzf5a.cloudfront.net/sites/default/files/podatsp.pdf

Nieva, G., Ortega, L. L., Mondon, S., Ballbè, M., Gual, A. (2010). Simultaneous versus delayed treatment of tobacco dependence in alcohol-dependent outpatients. Eur Addict Res 17:1-9

Olano, E., Minué, C., Ayesta, F.J. (2011). Tratamiento farmacológico de la dependencia de la nicotina: revisión de la evidencia científica y recomendaciones de la Sociedad Española de Especialistas en Tabaquismo sobre su elección e individualización. Med Clin., 136: 79-83.

Olmos, A., Tirado-Muñoz, J., Farré, M., Torrens, M. (2018) The efficacy of computerized interventions to reduce cannabis use: A systematic review and meta-analysis. Addictive Behaviors, 79, 52-60.

Peters, E. N., Budney, A.J., Carroll, K.M. (2012). Clinical correlates of co-occurring cannabis and tobacco use: a systematic review. Addiction 107(8): 1404-1417

Rabin, R. A. y George, T.P. (2015). A review of co-morbid tobacco and cannabis use disorders: possible mechanisms to explain high rates of co-use. The American Journal on Addiction 24: 105-116

Ramo, D. E., Liu, H., Prochaska, J.J. (2012). Tobacco and marijuana use among adolescents and young adults: a systematic review of their co-use. Clin Psychol Rev 32(2): 105-121

Sabioni, P. y Le Foll, B. (2018). Psychosocial and pharmacological interventions for the treatment of cannabis use disorder. F100OResearch 12 (7), 173. Disponible en 10.12688/f1000research.11191.1

Schwitzer, T., Gillet, C., Bisch, M., DiPatrizio, P., Schwan, R., Laprevote, V. (2016). Co-occurrent cannabis and tobacco uses: Clinical knowledge and therapeutic prospects. Thérapie, 71 (3), 315-322. Disponible en 10.1016/j.therap.2016.02.027

Sherman, B. J., McRae Clark, A. L. (2016). Treatment of Cannabis Use Disorder: Current Science and Future Outlook. Pharmacotherapy, 36, 511-535. Disponible en 10.1002/phar.1747

Spijkerman, R.J., Riper, R. (2013) Internet and computer based interventions for cannabis use: a meta-analysis. Drug and Alcohol Dependence, 133 (2),295-304.

Walther L., Gantner A., Heinz A., Maji T. (2016). Evidence based treatment options in cannabis dependency. Dtsch Arzteb/ Int , 113, 653-9. Disponible en 10.3238/arztebl.2016.0653

Whittaker, R., McRobbie, H., Bullen, C., Rodgers. A, Gu, Y. (2016) Mobile phone-based interventions for smoking cessation. Cochrane Database of Systematic Reviews 4 (CD006611). Disponible en 10.1002/14651858.CD006611. pub2

WHO (2011) mHealth: New horizons for health through mobile technologies: second global survey on eHealth. Disponible en https://www.who.int/goe/publications/goe_mhealth_web.pdf 